International Journal of Modern Physics A

(C) World Scientific Publishing Company

\title{
Theoretical Development on Pentaquarks
}

\author{
Shi-Lin Zhu \\ Department of Physics, Peking University, BEIJING 100871, CHINA
}

\begin{abstract}
I first give a brief overview of the status of exotic hadron search so far. Then I review the recent theoretical development on pentaquarks. Finally I emphasize that none of theoretical formalisms predicts the existence of $\Theta$ pentaquark very reliably. None of them explains its narrow width in a natural way.

Keywords: pentaquark, diquark
\end{abstract}

\section{Status of exotic hadron search}

Quark model is very successful in the classification of hadron states. Any state with quark content other than $q \bar{q}, q q q$ is beyond quark model. But quark model can't be the whole story. Quantum chromodynamics as the underlying theory of strong interaction allows much richer hadron spectrum such as: glueball, hybrid meson/baryon, multiquark states, hadron molecules etc.

In the meson sector, there are several non-conventional meson candidates. $f_{0}(980) / a_{0}(980)$ lies $10 \mathrm{MeV}$ below the $K^{+} K^{-}$threshold. It's difficult to find a suitable position for them within the framework of quark model. So they were postulated to be candidates of kaon molecule or four quark states. There are two hybrid meson candidates with exotic quantum numbers $J^{P C}=1^{-+}: \pi(1400), \pi(1600)$. However their masses are much lower than theoretical predictions from various approaches such as lattice QCD, QCD sum rule formalism and the flux tube model. Scalar glueballs may mix strongly with nearby scalar $q \bar{q}$ mesons. It's very demanding to establish the glueball candidates $f_{0}(1500), f_{0}(1700)$ experimentally although lattice QCD study indicates that pure scalar glueballs lie around $1500 \mathrm{MeV}$.

In the baryon sector, $\Lambda$ (1405) lies only $30 \mathrm{MeV}$ below the kaon nucleon threshold. It was postulated to be a kaon-nucleon molecule. Because of its low mass, Roper resonance was suggested to be a hybrid baryon with explicit glue inside. There does not exist a hybrid baryon with exotic quantum numbers. According to some models, the excitation of glue degree's of freedom carries an energy around $0.8 \mathrm{GeV}$ which renders the hybrid baryon to be much heavier than N(1440). Jaffe's H particle and other dibaryons candidates carry two units of baryon numbers. Till the end of 2002, NONE of these non-conventional hadron states has been established without controversy!

Since early 2003, there have been very important advances in hadron spec- 
troscopy. BES collaboration observed the $p \bar{p}$ and $p \Lambda$ near-threshold enhancement in $J / \Psi$ decays 1 . If these enhancements arise from a meson, they should also be observed in the mesonic final states 2] BABAR, CLEO and BELLE collaborations observed two narrow charm-strange mesons $D_{s J}(2317), D_{s J}(2457)$ below threshold 3 . These states may admit a small portion of $D K$ or $D^{*} K$ continuum contribution in their wave functions. But the dominant component of $D_{s J}(2317), D_{s J}(2457)$ should be $c \bar{s}$ प BELLE collaboration discovered a new narrow charmonium state $\mathrm{X}(3872)$ [5] Its production rate is comparable to that of other excited charmonium states. Some people suggested that it could be a deuteron-like $D \bar{D}^{*}$ molecule with four constituent quarks. Then it's very difficult to explain its big production rate $\underline{6}$. Recently SELEX collaboration reported a narrow state $D_{s J}(2632)$ above threshold with abnormal decay pattern 7 . Possible interpretations were suggested in Refs. $\underline{8}$. The most important event is LEPS and other collaborations reported evidence of pentaquarks 9 . A recent review can be found in Ref. 10.

\section{Pentaquarks: discovery or artifact}

Since last year LEPS and many other collaborationx reported the $\Theta^{+}$pentaquark at $1.54 \pm 0.01 \mathrm{GeV}$ with a width smaller than $25 \mathrm{MeV}$. Such a resonance with $B=+1, S=+1$ and the minimum quark content $u u d d \bar{s}$ is clearly beyond the conventional quark model. In the 2004 PDG $\Theta^{+}$has been listed as a three-star resonance.

However, many other experimental groups reported negative results 11. One may wonder how significant these negative experiments are. Some of them didn't see $\Sigma^{*}(1670), \Sigma^{*}(1660)$, which are four or three-star states in PDG! Certainly we can not rule out the existence of them based on the non-observation of these negative experiments. Moreover, production mechanism could be different for pentaquarks at high and low energy!

For those positive experiments, the following discrepancy should be clarified. $\Theta$ mass extracted from $n K^{+}$final states differs significantly from that extracted from the $p K_{s}$ channel. The width of $\Theta$ from direct measurements by HERMES and ZEUS groups is around ten $\mathrm{MeV}$, which is much larger than the upper bound set by the indirect analysis of kaon nucleus scattering data. Readers should keep in mind that the existence of $\Theta$ is still not completely established.

\section{Theoretical Developments on Pentaquarks}

If $\Theta$ really exists, there are serious theoretical challenges in the understanding of its low mass, very narrow width and weird production mechanism. In the following I list the main results of some theoretical approaches. I will not cover topics such as (1) non-clustered quark models; (2) pentaquark production; (3) $\Theta$ relatives; (4) heavy pentaquarks etc. 


\subsection{Lattice $Q C D$}

Lattice QCD starts from the first principle and should be the most reliable approach in the hadron spectroscopy. However the present status of lattice QCD calculation of pentaquark mass and parity is very confusing. I collect the available lattice results in the following table. Four groups observed pentaquark-like signals with negative parity on the lattice. One of them observed positive parity $\Theta$ pentaquark.

\begin{tabular}{|c|c|c|c|}
\hline Groups & Signal & Parity & Operators \\
\hline Csikor et al. 12 & Yes & Negative & Color-Octet Kaon Nucleon \\
Sasaki 13 & Yes & Negative & Diquark-Diquark-Anti-quark \\
Kentucky 14 & No & Negative & Color-Singlet Kaon Nucleon \\
Chiu-Hsieh 15 & Yes & Positive & Diquark-Diquark-Anti-quark \\
MIT 16 & Yes & Negative & Diquark-Diquark-Anti-quark \\
Ishii et al. 17 & No & Not Positive & Diquark-Diquark-Anti-quark \\
Takahashi et al. 18 & Yes & Negative & Color-Singlet(-Octet) KN \\
\hline
\end{tabular}

\subsection{Chiral soliton model and large $N_{c}$ expansion}

Diakanov et al. predicted $\Theta$ pentaquark at $1530 \mathrm{MeV}$ in the chiral soliton model (CSM) with $S=+1, B=1, J^{P}=\frac{1}{2}^{+}, I=0, \Gamma_{\Theta}<15 \mathrm{MeV}$ 19. They assumed $\mathrm{N}(1700)$ as a member of the anti-decuplet and used its mass and width as the benchmark to fix $\Theta$ mass and width. Recently CSM was updated by Ellis et al 20. With reasonable parameters, there always exists a low-lying $\Theta$ pentaquark: $1430<m_{\Theta}<1660 \mathrm{MeV}$. The anti-decuplet spectrum can be reproduced with bigger value of $\sigma$-term. The narrow width arises from the cancellation of the coupling constant in the first three orders in the large $N_{c}$ expansion.

But collective quantization may be invalid for pentaquarks in CSM 21. In this model, the mass splitting for non-exotic excitation is $\mathcal{O}\left(1 / N_{c}\right)$. For exotic excitaions, it is $\mathcal{O}\left(N_{c}^{0}\right)$. For pentaquarks, collective (rotation) and vibration modes are not orthogonal. They mix at the leading order of $N_{c}$ due to Wess-Zumino term. Collective quantization is NOT legitimate for exotic motion in CSM. The successful prediction of $\Theta$ properties in CSM is fortuitous 21 .

Large $N_{c}$ formalism does NOT predict $\Theta$ pentaquark. However, given the existence of $\Theta$, one can predict its large Nc partners in the same way that Delta is the $N_{c}$ partner of nucleon 22. The mass splitting between these states is $\mathcal{O}\left(1 / N_{c}\right)$. QCD consistency can be applied to scattering amplitudes to derive relations between coupling constants etc.

\subsection{QCD sum rules}

Similarly, QCD sum rules (QSR) does NOT predict $\Theta$ ! Instead, if it really exists, QSR can accommodate $\Theta$ at $1530 \mathrm{MeV}$ and yield the absolute mass scale without fit to data 23$]$. All QSR calculations 232425$]$ receive continuum contamination because 
of the high dimension of five-quark interpolating currents. QSR with old-fashion perturbation approach favors negative parity for $\Theta 24$. But the slow convergence of OPE series in this case is a serious problem 26. Appropriate interpolating currents such as color-octet KN type, diquark-diquark type can minimize the coupling with the scattering states as indicated by lattice QCD.

\subsection{Diquark model}

Jaffe and Wilczek proposed the diquark model for pentaquarks 27. They assumed $\Theta$ is composed of one $\bar{s}$ and a pair of $J^{P}=0^{+}, \overline{3}_{F}, \overline{3}_{c}$ [ud] diquark. Diquarks obey Bose statistics. Within their model, there exist an antidecuplet and octet with $J^{P}=\frac{1}{2}^{+}, \frac{3}{2}^{+}$. They are nearly degenerate. After ideal mixing, the lowest state is nucleon-like, which is identified as Roper resonance. But it is hard to dynamically generate the scalar diquark with a low mass $420 \mathrm{MeV}$ even with models. It's difficult to accommodate $\Theta$ and $\Theta_{c}(3099)$ simultaneously. Possible reasons could be (1) the scalar diquark mass is too low; (2) anti-quark related attraction is important and ignored in the diquark model. Some lattice simulations reproduced N(1440) using three quark interpolating currents, supporting Roper as nucleon's first radial excitation. $\mathrm{N}(1440)$ has a width of $350 \mathrm{MeV}$ while the upper bound of $\Theta$ width is $1 \mathrm{MeV}$. It's really hard to accommodate them simultaneously. Introduction of diquarks leads to many additional low-mass states beyond quark model such as four-quark states composed of a pair of diquark and anti-diquark. Where are they?

There exists $J^{P}=\frac{1}{2}^{-}$pentaquarks in diquark Model 28129. When the flavor wave function of two diquarks is anti-symmetric, one gets an octet and singlet with $L=0, J^{P}=\frac{1}{2}^{-}$. In other words, there are nine additional pentaquark states lighter than $\Theta$, close to the orbitally excited three-quark states. Especially two additional $\Lambda$-like pentaquarks lie around $\Lambda(1405)$. $\Lambda(1405)$ could be a pentaquark candidate! Two nucleon-like pentaquarks may be narrow. The lowest heavy pentaquark has $J^{P}=\frac{1}{2}^{-}$instead of $J^{P}=\frac{1}{2}^{+}$. Experimental discovery of these states is a direct support of the diquark model. Otherwise, one should re-evaluate the relevance of this picture.

The diquark and anti-quark are very similar in many respects. There exists a supersymmetry between diquark and anti-quark 3334 . If the diquark model is correct, pentaquarks can be related to anti-baryons through replacing two diquarks by two anti-quarks. Replacing the anti-quark by the diquark, pentaquarks can be related to dibaryons composed of three diquarks. With the experimental constraint on $\mathrm{H}$ dibaryon, one can estimate $\mathrm{P}=-S U_{F}(3)$ singlet pentaquark mass to be around(14021542) $\mathrm{MeV}$. With experimental $\Theta$ mass, $\mathrm{P}=$ - dibaryon mass is found to be around (2270-2310) $\mathrm{MeV}$ 33.

\subsection{Other clustered quark models}

There are other clustered quark models. Karliner and Lipkin proposed the diquarktriquark model [30] Two clusters were kept apart by the $\mathrm{P}$-wave angular momentum 
barrier. Color magnetic interaction occurs within two clusters only. Color electric forces bind them into a color singlet with $\mathrm{P}=+$. There exist an anti-decuplet and octet. In order to get a low lying $\Theta$, they assumed orbital excitation to be only 200 $\mathrm{MeV}$ to set scale. If one uses the realistic value of the spin-average orbital excitation around $500 \mathrm{MeV}, \Theta$ mass should be around $1800 \mathrm{MeV}$ in this model as pionted out by Hogaasen and Sorba 31 .

Shuryak and Zahed suggested replacing one scalar diquark in JW's model by a tensor one would lower $\Theta$ mass 32 . There is one orbital excitation within the tensor diquark. No orbital excitation between two diquarks is needed. But now $\Theta$ is in the 27 representation! One may wonder where $\Theta$ 's isospin partners are.

\section{Narrow width puzzle}

Around $\Theta$ mass region, $\Lambda(1520)$ decays via $\mathrm{D}$-wave into $\mathrm{K} \mathrm{N}$. Its width is $7 \mathrm{MeV}$. $\Lambda(1600)$ decays via $\mathrm{P}$-wave. Its width is $100 \mathrm{MeV}$. Both decays need the $q \bar{q}$ pair creation from vacuum. In contrast, $\Theta$ falls apart easily with similar phase space. But its width is less than $10 \mathrm{MeV}$ from direct measurements and less than $1 \mathrm{MeV}$ from indirect constraints. This is a big puzzle.

There are some attempts for narrow width puzzle including: (1) Color flavor spin overlap suppression 2713536, (2) Spatial overlap suppression 37, (3) Cancellation of coupling constants in the first three orders in the large NC expansion in CSM 1920; (4) Decoupling from the decay modes through the diagonalization of the mixing mass matrix between two degenerate pentaquarks 38; (5) Special diamond spatial structure 39 .

\section{Summary}

If $\Theta$ is confirmed and established by future experiments, a new landscape of multiquark hadrons is emerging from the horizon! We MUST answer: What's the underlying dynamics leading to its low mass, narrow width and special production mechanism? Do other multiquark hadrons exist: 4q, 6q, 7q, $\cdots, \mathrm{N}$ q? Is there an upper limit for N? Study of these issues will deepen our understanding of the lowenergy sector of QCD!

At present, it is fair to say that NONE of theoretical models predicts the existence of $\Theta(1530)$ reliably. NONE of theoretical models naturally explains its low mass, narrow width and production mechanism convincingly if it really exists. The spin and parity of $\Theta$ distinguish theoretical models. Now its existence is an experimental issue, requiring high-statistics experiments.

\section{Acknowledgments}

This project was supported by the National Natural Science Foundation of China under Grant 10375003, Ministry of Education of China, FANEDD and SRF for ROCS, SEM. 


\section{References}

1. J. Z. Bai et al, Phys. Rev. Lett. 91, 022001 (2003).

2. C-S. Gao, S.-L. Zhu, hep-ph/0308205 Commu. Theo. Phys. (in press).

3. B. Aubert et al., Phys. Rev. Lett. 90, 242001 (2003); D. Besson et al., Phys. Rev. D68, 032002 (2003); Y. Mikami et al., Phys. Rev. Lett. 92, 012002 (2004).

4. Y.-B. Dai, C.-S. Huang, C. Liu, S.-L. Zhu, Phys. Rev. D 68, 114011 (2003) and references therein.

5. S. K. Choi et al., Phys. Rev. Lett. 91, 262001 (2003).

6. Private communication with K.-T. Chao.

7. A. V. Evdokimov et al., hep-ex/00406045.

8. Y.-R, Liu et al., hep-ph/0407157 Phys. Rev. D (in press); Y.-B. Dai et al., hep-ph/0408234 and references therein.

9. T. Nakano et al., Phys. Rev. Lett. 91, 012002 (2003); V. V. Barmin et al., hep-ex/0304040 S. Stepanyan et al., hep-ex/0307018 J. Barth et al., hep-ph/0307083 A. E. Arataynet al., hep-ex/0309042 V. Kubarovsky et al., hep-ex/0311046 A. Airapetian et al., hep-ex/0312044 A. Aleev et al., hep-ex/0401024 COSY-TOF Collaboration, hep-ex/0403011 P. A. Aslanyan et al., hep-ex/0403044 ZEUS Collaboration, hep-ex/0403051| S. V. Chekanov, hep-ex/0404007

10. S.-L. Zhu, hep-ph/0406204 Int. J. Mod. Phys. A19, 3439 (2004).

11. J.Z. Bai et al., hep-ex/0402012 K. T. Knopfle et. al., hep-ex/0403020 C. Pinkenburg, nucl-ex/0404001

12. F. Csikor et al., hep-lat/0309090

13. S. Sasaki, hep-lat/0310014

14. N. Mathur et al., hep-ph/0406196

15. T.-W. Chiu, T.-H. Hsieh, hep-ph/0403020

16. MIT Collaboration, Talk at Quark Nuclear Physics 2004.

17. N. Ishii et al., hep-lat/0408030

18. F. Okiharu et al., hep-lat/0407001

19. D. Diakonov et al., Z. Phys. A 359, 305 (1997).

20. J. Ellis, M. Karliner and M. Praszalowicz, hep-ph/0410127

21. T. D. Cohen, hep-ph/0309111 hep-ph/0312191 N. Itzhaki et al., hep-ph/0309305

22. T. D. Cohen, hep-ph/0402056 E. Jenkins, A. V. Manohar, hep-ph/0401190 0402024, 0402150.

23. S.-L. Zhu, Phys. Rev. Lett. 91, 232002 (2003).

24. J. Sugiyama et al., hep-ph/0309271

25. R.D. Matheus et al., hep-ph/0309001

26. Private communications with Kim Maltman.

27. R. Jaffe and F. Wilczek, Phys. Rev. Lett. 91, 232003 (2003).

28. A. Zhang et al., hep-ph/0403210

29. I. Stewart et al., hep-ph/0402076

30. M. Karliner and H. J. Lipkin, hep-ph/0307243

31. H. Hogaasen and P. Sorba, hep-ph/0406078

32. E. Shuryak and I. Zahed, hep-ph/0310270

33. S.-L. Zhu, hep-ph/0405149 Phys. Rev. C (in press).

34. D. B. Lichtenberg, hep-ph/0406198

35. B.Jennings and K.Maltman, hep-ph/0308286

36. F. E. Close and J. J. Dudek, hep-ph/0401192

37. D. Melikhov et al., Phys.Lett.B594, 265 (2004).

38. M. Karliner and H. J. Lipkin, hep-ph/0410072

39. X.-C. Song and S.-L. Zhu, hep-ph/0403093 Mod. Phys. Lett. A (in press). 